Article

\title{
Time-Dependent Wave-Structure Interaction Revisited: Thermo-Piezoelectric Scatterers ${ }^{\dagger}$
}

\author{
George C. Hsiao ${ }^{1}$ and Tonatiuh Sánchez-Vizuet ${ }^{2, *(D)}$ \\ 1 Department of Mathematical Sciences, University of Delaware, Newark, DE 19716, USA; ghsiao@udel.edu \\ 2 Department of Mathematics, The University of Arizona, Tucson, AZ 85721, USA \\ * Correspondence: tonatiuh@math.arizona.edu \\ + Dedicated to Professor Wolfgang L. Wendland on the occasion of his 85th Birthday.
}

Citation: Hsiao, G.C.;

Sánchez-Vizuet, T. Time-Dependent Wave-Structure Interaction Revisited: Thermo-Piezoelectric Scatterers. Fluids 2021, 6, 101. https://doi.org/ $10.3390 /$ fluids 6030101

Academic Editor: Iman Borazjan

Received: 10 February 2021

Accepted: 26 February 2021

Published: 3 March 2021

Publisher's Note: MDPI stays neutra with regard to jurisdictional claims in published maps and institutional affiliations.

Copyright: (c) 2021 by the authors. Licensee MDPI, Basel, Switzerland This article is an open access article distributed under the terms and conditions of the Creative Commons Attribution (CC BY) license (https:/ / creativecommons.org/licenses/by/ $4.0 /)$.
Abstract: In this paper, we are concerned with a time-dependent transmission problem for a thermopiezoelectric elastic body that is immersed in a compressible fluid. It is shown that the problem can be treated by the boundary-field equation method, provided that an appropriate scaling factor is employed. As usual, based on estimates for solutions in the Laplace-transformed domain, we may obtain properties of corresponding solutions in the time-domain without having to perform the inversion of the Laplace-domain solutions.

Keywords: wave-structure interaction; coupling procedure; kirchhoff representation formula; retarded potential; laplace transform; boundary integral equation; variational formulation; sobolev space; transient waves; thermoelasticity; piezoelectricity

MSC: 35J20; 35L05; 45P05; 65N30; 65N38

\section{Introduction}

The mathematical description of the interaction between an acoustic wave and an elastic body is of central importance in applied mathematics and engineering, as attested, for instance, by its usage for the detection and identification of submerged objects. The problem is mathematically formulated as a transmission problem between elastic and acoustic fields communicating through an interface and it is referred to in the literature either as "fluid-structure interaction problem" or "wave-structure interation problem". The former terminology (wave-structure interaction) is also used to describe a similar problem that involves the coupling between fluid equations (either Stokes or Navier-Stokes) and the equations of elasticity. Here, we will be interested in the coupling between the acoustic and elastic wave equations, and we will use the term "wave-structure interaction" exclusively to avoid any confusion.

In the early days of the field, most of the mathematical formulations of these kinds of problems were based on time-harmonic formulations. Being motivated by the paper of Mamdi and Jean [1], Hsiao, Kleinman, and Schuetz 's paper from 1988 [2] gave the first mathematical justification of a variational formulation for wave-structure interaction problems. This set out the field for many further efforts that expanded the understanding of time-harmonic scattering (see, e.g., [3-7]). Over the years, time-harmonic wave-structure interaction problems have been studied in various different areas, such as inverse problems [8,9], interaction of fluid and thin structures [10], and interaction of electromagnetic fields and elastic bodies $[11,12]$, just to name a few.

One of the main reasons behind the use of the boundary-field equation method for treating time-harmonic wave-structure problems is to reduce the transmission problem, posed originally in an unbounded domain, to one set in the bounded domain $\Omega$ that was determined by the elastic scatterer (see Figure 1). However, the conversion from an unbounded to a bounded domain comes at the price of turning the problem into a non-local 
one, which brings along some mathematical disadvantages. Because the sesquilinear form arising from the nonlocal boundary problem can only satisfy a Gårding inequality, in order to apply the standard Fredholm alternative for the existence theory, the uniqueness of the solution becomes a requirement. However, the straightforward boundary-field method can not circumvent the drawbacks, because the problem is not uniquely solvable when the frequency of the incident wave coincides with what is known as a "Jones frequency". At such a frequencies, the corresponding homogeneous problem may have traction free solutions (a recent discussion on this can be found in [13]). Moreover, the uniqueness of the solutions to the boundary integral equations may not be guaranteed when the exterior wavenumber coincides with an eigenvalue of the corresponding interior Dirichlet problem (see [14]). The issue of non-uniqueness has motivated lots of research, and attempts to overcome these difficulties have been made with the help of methods, such as Schenck's Combined Helmholtz Integral Equation Formulation [15] (commonly known as the CHIEF method) and the celebrated formulation by Burton and Miller [16].

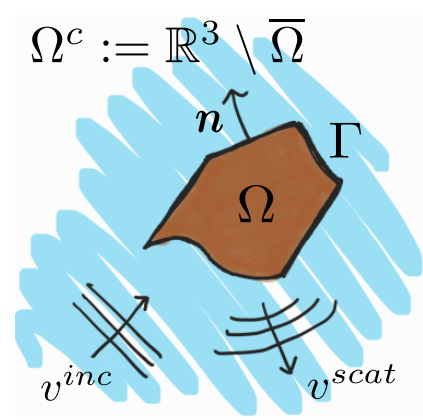

Figure 1. Schematic of the wave scattering problem. The interface between the solid and the fluid is denoted by $\Gamma$, while the outward-pointing normal vector (that is defined for almost every point in the boundary) is denoted by $\boldsymbol{n}$.

In the present paper, being inspired by the work of Estorff and Antes [17], we will apply the boundary-field equation method not to a time-harmonic problem, but rather one in the transient regime. This will require the treatment of the wave equation, as opposed to the Helmholtz equation that is used in the frequency domain. The problem of interest is that of the interaction between a thermo-piezoelectric elastic body that is immersed in a compressible fluid. The method will not be directly applied in the time-domain, but rather in the Laplace transformed domain. The reasons for this will be made clear in due time. The equations will then be reduced to those of a nonlocal boundary problem in the transformed domain, where all tof he analysis will be performed. The technique that will be applied will allow for us to understand the behavior of the transient problem (and even simulate it computationally if we were so inclined) without ever having to invert the Laplace transform.

The outline of the solution/analysis procedure for the time-dependent wave-structure interaction is as follows:

1. Formulate a time-dependent transmission problem.

2. Apply the Laplace transform to the time-dependent transmission problem.

3. Reduce the transformed transmission problem to a nonlocal boundary problem in the bounded domain $\Omega$ with the help of a Boundary Integral Equation (BIE). This leads to the boundary-field equation formulation of the problem in the transformed domain.

4. Obtain estimates of variational solutions of the nonlocal boundary problem in terms of the Laplace transformed variable $s$.

5. Deduce estimates for the solutions in the time domain from those of the corresponding solutions in the Laplace domain while using Lubich's and Sayas's approach for treating BIEs of the convolution type [18-20]).

The process that is described above has been successfully applied to a number of special cases [21-24]. However, in all of the cases under consideration, the formulations 
in the fluid domain were given in terms of velocity potentials, not in terms of standard fluid pressures. As will be seen, an appropriate scaling factor will have to be introduced to formulate the problem in term of fluid pressure.

The analysis will proceed more or less following the steps that are outlined above. Section 2 introduces the time-domain formulation of the problem, and Section 3 then describes the corresponding nonlocal boundary problem in the Laplace transferred domain. Section 4 contains mathematical ingredients concerning crucial estimates for the solution of the nonlocal boundary problem in the transformed domain. Section 5 presents the main results in the time domain and Section 6 ends the paper with some concluding remarks.

\section{Formulations of the Problem}

We will denote by $\Omega$ an open and bounded subset of $\mathbb{R}^{3}$ that will be considered to be occupied by an elastic solid. We will further assume that the boundary of the solid is described by a Lipschitz-continuous curve and it will be denoted by $\Gamma$. The exterior of this solid, which will be denoted by $\Omega^{c}=\mathbb{R}^{3} \backslash \bar{\Omega}$, will be filled by an inviscid and compressible fluid. Figure 1 depicts a schematic of the geometric setting.

We will consider that, when at rest, the velocity, pressure, and density in the fluid are described by the constant fields $\mathbf{v}_{0}=0, p_{0}$, and $\rho_{f}$, and will be interested in the time evolution of small parturbations from this static configuration, as described by the fields $\mathbf{v}, p$ and $\rho$, which is given by the linearized Euler equation in the fluid domain $\Omega^{c}$

$$
\rho_{f} \frac{\partial \mathbf{v}}{\partial t}+\nabla p=\mathbf{0}
$$

the continuity equation

$$
\frac{\partial \rho}{\partial t}+\rho_{f} \nabla \cdot \mathbf{v}=0
$$

for $\rho$, and $\mathbf{v}$, and the state equation for $p$ and $\rho$

$$
p=c^{2} \rho .
$$

Above, the sound speed $c$ is a function that varies depending on the properties of the fluid (see e.g., $[25,26]$ ), and the operator $\frac{\partial}{\partial t}$ is the usual partial derivative with respect to the time variable, not to be confused with the material derivative. All of these equations are posed in $\Omega^{c} \times[0, \infty)$. A simple manipulation shows that, with the help of Equations (2) and (3), we may replace Equation (1) by a single wave equation for the pressure $p$

$$
\frac{1}{c^{2}} \frac{\partial^{2} p}{\partial t^{2}}-\Delta p=0 \quad \text { in } \quad \Omega^{c} \times[0, \infty)
$$

Now, inside the domain $\Omega$ that is occupied by the solid, the governing equation depends on the properties of the solid. It may be as simple as an elastic obstacle, or it may have more complicated physical properties, such as a thermoelastic solid, or a thermopiezoelectric solid, as in our present case. The problem under consideration, for a thermo-piezoelectric body, consists of determining the stress and strains tensors, $\sigma(x, t)$ and $\varepsilon(x, t)$, the elastic displacement $\mathbf{u}(x, t)$, temperature variation $\theta(x, t)$, and the electric potential $\varphi(x, t)$. The physics of the process can be described in terms of the reference density of the solid $\rho_{e}$, the absolute temperature in the solid $T$ and its stress-free reference temperature $T_{0}$, the electric displacement vector $\mathbf{D}(x, t)$, and the entropy per unit volume $P(x, t)$. The governing equations have been derived by Mindlin [27] and they consist of three coupled partial differential equations, namely the dynamic elastic equations:

$$
\rho_{e} \frac{\partial^{2} \mathbf{u}}{\partial t^{2}}-\nabla \cdot \sigma=\mathbf{0},
$$


the generalized heat equation

$$
T \frac{\partial P}{\partial t}-\Delta \theta=0, \quad \theta:=T-T_{0}
$$

and the equation of the quasi-stationary electric field (i.e., Gauss's electric field law without electric charge density):

$$
\nabla \cdot \mathbf{D}=0 .
$$

These equations need to be supplied with adequate constitutive relations providing a description of the functional dependence between the unknown variables within the thermo-piezoelectric media. In the isotropic case, the constitutive relations may be simplified in the form (see [28]):

$$
\begin{aligned}
& \sigma=\sigma(\mathbf{u}, \theta, \varphi):=\sigma_{e}(\mathbf{u})-\zeta \theta \mathbf{I}-\mathbf{e}^{\top} \mathbf{E} \\
& P=P(\mathbf{u}, \theta, \varphi):=\zeta \nabla \cdot \mathbf{u}+\frac{c_{\epsilon}}{T_{0}} \theta+\mathbf{p} \cdot \mathbf{E} \\
& \mathbf{D}=\mathbf{D}(\mathbf{u}, \theta, \varphi):=\mathbf{e} \varepsilon(\mathbf{u})+\theta \mathbf{p}+\epsilon \mathbf{E}
\end{aligned}
$$

where:

$$
\sigma_{e}:=\lambda(\nabla \cdot \mathbf{u}) \mathbf{I}+2 \mu \varepsilon(\mathbf{u}), \quad \text { and } \quad \varepsilon(\mathbf{u}):=\frac{1}{2}\left(\nabla \mathbf{u}+\nabla \mathbf{u}^{\top}\right)
$$

are the usual stress and strain tensors for isotropic elastic media, while $\mathbf{e}=\left(\left(\mathbf{e}_{i j k}\right)\right)$ is the piezoelectric tensor with constant elements, such that $\mathrm{e}_{k i j}=\mathrm{e}_{k j i}$. This third order tensor maps matrices into vectors, while its adjoint, which will be denoted by $\mathbf{e}^{\top}$, maps vectors into symmetric matrices. More precisely, for a real symmetric matrix $\mathbf{M} \in \mathbb{R}_{s y m}^{d \times d}$ and for a vector, $\mathbf{d} \in \mathbb{R}^{d}$, we define:

$$
(\mathbf{e M})_{k}:=\sum_{i j} \mathrm{e}_{k i j} \mathbf{M}_{i j} \in \mathbb{R}^{d} \quad \text { and } \quad\left(\mathbf{e}^{\top} \mathbf{d}\right)_{i j}:=\sum_{k} \mathrm{e}_{k i j} \mathbf{d}_{k} \in \mathbb{R}_{s y m}^{d \times d} .
$$

The constants $\zeta$ and $\epsilon$ are, respectively, the thermal and dielectric constants; $c_{\epsilon}$ is the specific heat at constant strain, and the constant vector $\mathbf{p}$ is the pyroelectric moduli vector. The electric field $\mathbf{E}$ in the constitutive equations is replaced by $\mathbf{E}=-\nabla \varphi$. As usual, $\mu>0$ and $\lambda$ are the Lamé constants for the elastic body (note that it is customary to require $\lambda>0$, however this is not necessary as long as the physically meaningful quantity $3 \lambda+2 \mu$, which is known as the bulk modulus, remains positive). Mindlin [27] first proposed the theory of thermopiezoelectricity. The physical laws for thermopiezoelectric materials were explored by Nowacki [29] (GCH would like to thank Prof. T.W. Chou for locating this reference for him) [30], where more general constitutive relations are available than those that are given in Equations (7) and (8).

Making use of these constitutive relations in conjunction with the governing Equations (4)-(6), we arrive at differential equations.

$$
\begin{aligned}
\rho_{e} \frac{\partial^{2} \mathbf{u}}{\partial t^{2}}-\nabla \cdot\left(\sigma_{e}(\mathbf{u})-\zeta \theta \mathbf{I}+\mathbf{e}^{\top} \nabla \varphi\right) & =\mathbf{0} \\
\frac{\partial}{\partial t}(\zeta \nabla \cdot \mathbf{u}-\mathbf{p} \cdot \nabla \varphi)+\frac{1}{T_{0}}\left(c_{\epsilon} \frac{\partial \theta}{\partial t}-\Delta \theta\right) & =0 \\
\nabla \cdot(\mathbf{e} \varepsilon(\mathbf{u})+\theta \mathbf{p}-\epsilon \nabla \varphi) & =0
\end{aligned}
$$

We remark that Equation (10) is derived under the assumption that $\left|\frac{\theta}{T_{0}}\right| \ll 1$. This means $T \simeq T_{0}$, since $T=T_{0}\left(1+\frac{\theta}{T_{0}}\right)$. Equations (9)-(11) constitute the complete set of equations of thermopiezoelectricity coupling a hyperbolic equation for $\mathbf{u}$, a parabolic equation for $\theta$, and an elliptic equation for $\varphi$. Here and in the sequel, all of the constant physical quantities satisfy:

$$
\rho_{e}>0, \mu>0,3 \lambda+2 \mu>0, \mathrm{e}_{i j k}>0, \zeta>0, c_{\varepsilon}>0 .
$$


In order to formulate a typical time-dependent wave-structure problem, we need to prescribe initial, boundary, and transmission conditions. This leads to a model of partial differential equations for the time-dependent wave-structure problem.

Time-dependent transmission problem. Given $\left(p^{i n c}, \partial_{n} p^{i n c}, f_{\theta}, f_{\mathbf{D}}\right)$, find the solutions $(\mathbf{u}, \theta, \varphi)$ in $\Omega \times[0, \infty)$, and $p$ in $\Omega^{c} \times[0, \infty)$ satisfying the partial differential equations

$$
\begin{aligned}
& \rho_{e} \frac{\partial^{2} \mathbf{u}}{\partial t^{2}}-\nabla \cdot\left(\sigma_{e}(\mathbf{u})-(\zeta \theta) \mathbf{I}+\mathbf{e}^{\top} \nabla \varphi\right)=\mathbf{0} \quad \text { in } \quad \Omega \times[0, \infty) \\
& \frac{\partial}{\partial t}(\zeta \nabla \cdot \mathbf{u}-\mathbf{p} \cdot \nabla \varphi)+\frac{1}{T_{0}}\left(c_{\epsilon} \frac{\partial \theta}{\partial t}-\Delta \theta\right)=0 \quad \text { in } \quad \Omega \times[0, \infty) \\
& \nabla \cdot(\mathbf{e} \varepsilon(\mathbf{u})+\theta \mathbf{p}-\epsilon \nabla \varphi)=0 \quad \text { in } \quad \Omega \times[0, \infty)
\end{aligned}
$$

and

$$
\frac{1}{c^{2}} \frac{\partial^{2} p}{\partial t^{2}}-\Delta p=0 \quad \text { in } \quad \Omega^{c} \times[0, \infty)
$$

together with the transmission conditions

$$
\begin{aligned}
& \sigma(\mathbf{u}, \theta, \varphi)^{-} \mathbf{n}=-\left(p+p^{i n c}\right)^{+} \mathbf{n} \quad \text { on } \Gamma \times[0, \infty), \\
& \frac{\partial \mathbf{u}^{-}}{\partial t} \cdot \mathbf{n}=-\frac{1}{\rho_{f}} \int_{0}^{t} \frac{\partial}{\partial n}\left(p+p^{i n c}\right)^{+} d \tau \text { on } \Gamma \times[0, \infty),
\end{aligned}
$$

the boundary conditions

$$
\partial_{n} \theta=f_{\theta}, \quad \text { and } \mathbf{D} \cdot n=f_{\mathbf{D}} \text { on } \Gamma \times[0, \infty)
$$

and homogeneous initial conditions for $\mathbf{u}, \partial \mathbf{u} / \partial t, \theta, p$ and $\partial p / \partial t$.

The given data and solutions are required to satisfy certain regularity properties that will be specified later. In the formulation, we use the superscripts ${ }^{+}$or $^{-}$to denote the traces or restrictions to the boundary $\Gamma$ of a function when taken as limits from functions defined on $\Omega^{c}$ and $\Omega$, respectively. This is equivalent to the notation $v^{+}=\gamma^{+} v$ and $v^{-}=\gamma^{-} v$ customary in the mathematical literature. Whenever the trace-or restriction-of a function to the boundary does not depend on the side from which the limit is taken, we will drop the superscript and only write $\gamma v$. In this formulation, one has to solve the wave equation for the pressure in the exterior-unbounded-domain, which can be a drawback from the computational point of view.

In order to sidestep the challenge of undboundedness, we will resort to a formulation of the transmission problem that is defined by Equations (12)-(15) that will couple boundary integral equations with partial differential equations. This technique, put forward in the context of time-harmonic problems [14], transforms the problem into a nonlocal one that is only posed in the bounded computational domain $\Omega$ by representing the pressure in the fluid domain through an integral along the interface $\Gamma$ between the solid and the fluid. To this avail, we must introduce the fundamental solution to the wave equation:

$$
\mathcal{G}(x-y, t)=\frac{1}{4 \pi|x-y|} \delta\left(t-c^{-1}|x-y|\right) .
$$

Above, $\delta(\cdot)$ is Dirac's delta. Using this fundamental solution, it is possible to express any solution to Equation (13) in terms of density functions $\phi$, and $\lambda$ that correspond to the Cauchy data of the problem, namely, the pressure restricted to $\Gamma$ and its normal derivative, respectively. This is known as the Kirchhoff representation formula (see e.g., [18,31,32])

$$
p(x, t)=(\mathcal{D} * \phi)(x, t)-(\mathcal{S} * \lambda)(x, t), \quad(x, t) \in \Omega^{c} \times[0, \infty) .
$$


Above, the asterisk $*$ refers to convolution with respect to time,

$$
f * g=\int_{0}^{t} f(t-\tau) g(\tau) d \tau,
$$

and $\mathcal{D}$ and $\mathcal{S}$ are known, respectively, as the double- and simple-layer potentials. They can be defined as convolutions with the fundamental solution and its normal derivative:

$$
\begin{aligned}
(\mathcal{S} * \lambda)(x, t) & :=\int_{0}^{t} \int_{\Gamma} \mathcal{G}(\boldsymbol{x}-\boldsymbol{y}, t-\tau) \lambda(\boldsymbol{y}, \tau) d \Gamma_{y} d \tau \\
& =\int_{\Gamma} \frac{1}{4 \pi|\boldsymbol{x}-\boldsymbol{y}|} \lambda\left(\boldsymbol{y}, t-c^{-1}|\boldsymbol{x}-\boldsymbol{y}|\right) d \Gamma_{\boldsymbol{y}} \\
& =\int_{\Gamma} E(\boldsymbol{x}, \boldsymbol{y}) \lambda\left(\boldsymbol{y}, t-c^{-1}|\boldsymbol{x}-\boldsymbol{y}|\right) d \Gamma_{\boldsymbol{y}}, \\
(\mathcal{D} * \phi)(\boldsymbol{x}, t) & :=\int_{0}^{t} \int_{\Gamma} \frac{\partial}{\partial n_{y}} \mathcal{G}(\boldsymbol{x}-\boldsymbol{y}, t-\tau) \phi(\boldsymbol{y}, \tau) d \Gamma_{y} d \tau \\
& =\int_{\Gamma} \frac{\partial}{\partial n_{y}}\left(\frac{1}{4 \pi|x-y|} \phi\left(\boldsymbol{y}, t-c^{-1}|\boldsymbol{x}-\boldsymbol{y}|\right)\right) d \Gamma_{\boldsymbol{y}} \\
& =\int_{\Gamma} \frac{\partial}{\partial n_{y}}\left(E(\boldsymbol{x}, \boldsymbol{y}) \phi\left(\boldsymbol{y}, t-c^{-1}|\boldsymbol{x}-\boldsymbol{y}|\right)\right) d \Gamma_{y} .
\end{aligned}
$$

In these equations, we have denoted the fundamental solution of the negative Laplacian in $\mathbb{R}^{3}$ by $E(x, y):=\frac{1}{4 \pi|x-y|}$. The reader will notice that the convolution with the fundamental solution introduces a delay into the density functions $\lambda$ and $\phi$. It is customary in the wave propagation community, to write $[\varphi]=\varphi\left(\boldsymbol{y}, t-c^{-1}|\boldsymbol{x}-\boldsymbol{y}|\right)$ and call $[\varphi]$ the retarded value of $\varphi$. This is the reason why sometimes $(\mathcal{S} * \lambda)(\boldsymbol{x}, t)$ and $(\mathcal{D} * \phi)(\boldsymbol{x}, t)$ are referred to as the retarded layer potentials.

Similarly, by introducing the convolution integral:

$$
(\mathcal{I} * \varphi)(\boldsymbol{x}, t):=\int_{0}^{t} \int_{\Gamma} \delta(\boldsymbol{x}-\boldsymbol{y} ; t-\tau) \varphi(\boldsymbol{y}, \tau) d \Gamma_{\boldsymbol{y}} d \tau=\varphi(\boldsymbol{x}, t),
$$

At non-singluar points of $\Gamma$, the Cauchy data $\phi$ and $\lambda$ satisfy the following system of boundary integral equations (see, e.g., [33-36])

$$
\left(\begin{array}{l}
\phi \\
\lambda
\end{array}\right)=\left(\begin{array}{cc}
\frac{1}{2} \mathcal{I}+\mathcal{K} & -\mathcal{V} \\
-\mathcal{W} & \left(\frac{1}{2} \mathcal{I}-\mathcal{K}\right)^{\prime}
\end{array}\right) *\left(\begin{array}{l}
\phi \\
\lambda
\end{array}\right) \quad \text { on } \quad \Gamma \times[0, \infty) .
$$

The boundary integral operators $\mathcal{V}, \mathcal{K}, \mathcal{K}^{\prime}$, and $\mathcal{W}$ appearing above are known, respectively, as the simple layer, double layer, transpose double layer, and hypersingular boundary integral operators for the dynamic wave equation. They are defined, as follows:

$$
\left.\begin{array}{rl}
(\mathcal{V} * \lambda) & :=\{\{\gamma(\mathcal{S} * \lambda)\}\}=\frac{1}{2}\left(\gamma^{-}(\mathcal{S} * \lambda)+\gamma^{+}(\mathcal{S} * \lambda)\right) \\
& =\gamma^{-}(\mathcal{S} * \lambda)=\gamma^{+}(\mathcal{S} * \lambda) \\
(\mathcal{K} * \phi) & :=\{\{\gamma(\mathcal{K} * \phi)\}\}=\frac{1}{2}\left(\gamma^{-}(\mathcal{K} * \phi)+\gamma^{+}(\mathcal{K} * \phi)\right) \\
\left(\mathcal{K}^{\prime} * \lambda\right) & :=\left\{\left\{\gamma\left(\mathcal{K}^{\prime} * \lambda\right\}\right\}=\frac{1}{2}\left(\gamma^{-}\left(\mathcal{K}^{\prime} * \lambda\right)+\gamma^{+}\left(\mathcal{K}^{\prime} * \lambda\right)\right)\right. \\
(\mathcal{W} * \phi) & :=-\left\{\left\{\partial_{n}(\mathcal{D} * \phi)\right\}\right\}=-\frac{1}{2}\left(\partial_{n}^{-}(\mathcal{D} * \lambda)+\partial_{n}^{+}(\mathcal{D} * \lambda)\right) \\
& =-\partial_{n}^{-}(\mathcal{D} * \phi)=-\partial_{n}^{+}(\mathcal{D} * \lambda)
\end{array}\right\} \text { on } \quad \Gamma \times[0, \infty)
$$


Note that the averaging operator $\{\{\cdot\}\}$ has been implictly defined in the second equality on the first line above.

We can now state the reformulation of the original problem that we will be focusing on:

Time-dependent nonlocal problem. Given $\left(p^{i n c}, \partial_{n} p^{i n c}, f_{\theta}, f_{\mathbf{D}}\right)$, find the solutions $(\mathbf{u}, \theta, \varphi)$ in $\Omega \times[0, \infty)$ and $(\phi, \lambda)$ on $\Gamma \times[0, \infty)$ satisfying the partial differential equations

$$
\begin{aligned}
\rho_{e} \frac{\partial^{2} \mathbf{u}}{\partial t^{2}}-\nabla \cdot\left(\sigma_{e}(\mathbf{u})-(\gamma \theta) \mathbf{I}+\mathbf{e}^{\top} \nabla \varphi\right) & =\mathbf{0} \quad \text { in } \quad \Omega \times[0, \infty), \\
\frac{\partial}{\partial t}(\gamma \nabla \cdot \mathbf{u}-\mathbf{p} \cdot \nabla \varphi)+\frac{1}{T}{ }_{0}\left(c_{\epsilon} \frac{\partial \theta}{\partial t}-\Delta \theta\right) & =0 \quad \text { in } \Omega \times[0, \infty), \\
\nabla \cdot(\mathbf{e} \varepsilon(\mathbf{u})+\theta \mathbf{p}-\epsilon \nabla \varphi) & =0 \quad \text { in } \quad \Omega \times[0, \infty),
\end{aligned}
$$

and the differential-boundary integral equations

$$
\begin{array}{cc}
-\rho_{f} \frac{\partial \mathbf{u}}{\partial t} \cdot \mathbf{n}+\int_{0}^{t}\left((\mathcal{W} * \phi)(x, t)-\frac{1}{2} \lambda(x, t)+\left(\mathcal{K}^{\prime} * \lambda\right)(x, t)\right) d \tau=\int_{0}^{t} \partial_{n}^{+} p^{i n c} d \tau & \text { on } \Gamma \times[0, \infty), \\
\frac{1}{2} \phi(x, t)-(\mathcal{K} * \phi)(x, t)+(\mathcal{V} * \lambda)(x, t)=0 & \text { on } \Gamma \times[0, \infty) .
\end{array}
$$

together with the transmission condition

$$
\boldsymbol{\sigma}(\mathbf{u}, \theta, \varphi)^{-} \mathbf{n}=-\left(\phi+p^{i n c}\right)^{+} \mathbf{n}, \quad \text { on } \Gamma \times[0, \infty),
$$

the boundary conditions

$$
\partial_{n} \theta=f_{\theta}, \quad \text { and } \mathbf{D} \cdot n=f_{\mathbf{D}} \quad \text { on } \Gamma \times[0, \infty),
$$

as well as homogeneous initial conditions for $\mathbf{u}, \partial \mathbf{u} / \partial t, \theta, \phi$ and $\lambda$.

Throughout the paper, the given data $\left(p^{i n c}, \partial_{n} p^{i n c}, f_{\theta}, f_{D}\right)$ will always be assumed to be causal functions, namely, functions of time $t$ that identically vanish for $t<0$.

From the definitions of the operators $\mathcal{V}, \mathcal{K}, \mathcal{K}^{\prime}$, and $\mathcal{W}$, we notice that the non-locality of the boundary integral equations in (17) is not restricted to space, but also extends into the time variable.

To study the well-posedness of this formulation, we will first transform it to the Laplace domain, where the analysis will be performed. This idea is due to Lubich and Schneider (see, e.g., $[19,20]$ ) and it has been extended by Laliena and Sayas $[18,37]$. We remark that the passage to the Laplace domain is only required to simplify the analysis and the stability estimates, but, for a computational implementation, this technique does not require the numerical inversion of the Laplace transform. Instead, from the estimates of the solutions in the transformed domain, the properties of the solutions in the time domain will be automatically deduced. The latter is particularly desirable from the computational point of view. In the next section, we will consider the model of partial differential equations for the time-dependent wave-structure problem and/or the time-dependent nonlocal boundary transmission problem in the Laplace domain.

\section{A Nonlocal Boundary Problem}

The passage to the Laplace domain will require us to first introduce some definitions. The complex plane be denoted in the sequel by $\mathbb{C}$, while we will use the notation

$$
\mathbb{C}_{+}:=\{s \in \mathbb{C}: \operatorname{Re} s>0\},
$$


to refer to the positive half plane. For any complex-valued function with limited growth at infinity $f:[0, \infty) \rightarrow \mathbb{C}$, its Laplace transform is given by

$$
\widehat{f}(s)=\mathcal{L} f(s):=\int_{0}^{\infty} e^{-s t} f(t) d t,
$$

whenever the integral converges. A broad class of functions for which the Laplace transform is well-defined is that of functions of exponential order. More precisely, a function $f$ is said to be of exponential order if there exist constants $t_{0}>0, M \equiv M\left(t_{0}\right)>0$, and $\alpha \equiv \alpha\left(t_{0}\right)>0$, satisfying:

$$
t \geq t_{0} \Longrightarrow|f(t)| \leq M e^{\alpha t}
$$

In the following, let $\widehat{\mathbf{u}}(s):=\mathcal{L}\{\mathbf{u}(x, t)\}, \widehat{\theta}(s):=\mathcal{L}\{\theta(x, t)\}, \widehat{\varphi}(s):=\mathcal{L}\{\varphi(x, t)\}$, and $\widehat{p}(s):=\mathcal{L}\{p(x, t)\}$. Subsequently, in the Laplace domain, Equations (12)-(14) become

$$
\begin{array}{r}
-\nabla \cdot\left(\sigma_{e}(\widehat{\mathbf{u}})-(\zeta \widehat{\theta}) \mathbf{I}+\mathbf{e}^{\top} \nabla \widehat{\varphi}\right)+\rho_{e} s^{2} \widehat{\mathbf{u}}=\mathbf{0} \quad \text { in } \quad \Omega \\
s(\zeta \nabla \cdot \widehat{\mathbf{u}}-\mathbf{p} \cdot \nabla \widehat{\varphi})+\frac{1}{T_{0}}\left(-\Delta \widehat{\theta}+c_{\epsilon} s \widehat{\theta}\right)=0 \text { in } \Omega \\
\nabla \cdot(\mathbf{e} \varepsilon(\widehat{\mathbf{u}})+\widehat{\theta} \mathbf{p}-\epsilon \nabla \widehat{\varphi})=0 \text { in } \Omega
\end{array}
$$

and

$$
-\Delta \widehat{p}+\frac{s^{2}}{c^{2}} \widehat{p}=0 \quad \text { in } \quad \Omega^{c} .
$$

together with the transmission conditions:

$$
\begin{gathered}
\sigma(\widehat{\mathbf{u}}, \widehat{\theta}, \widehat{\varphi})^{-} \mathbf{n}=-\left(\hat{p}+\hat{p}^{i n c}\right)^{+} \mathbf{n} \quad \text { on } \quad \Gamma, \\
s^{2} \widehat{\mathbf{u}} \cdot \mathbf{n}=-\frac{1}{\rho_{f}} \frac{\partial}{\partial n}\left(\widehat{p}+\hat{p}^{i n c}\right)^{+} \text {on } \Gamma,
\end{gathered}
$$

and the boundary conditions:

$$
\partial_{n} \widehat{\theta}=\widehat{f}_{\theta}, \quad \text { and } \quad \widehat{\mathbf{D}} \cdot n=\widehat{f}_{\mathbf{D}} \quad \text { on } \quad \Gamma .
$$

Above, analogously to the time-domain system, the generalized stress tensor is given by $\sigma(\widehat{\mathbf{u}}, \widehat{\theta}, \widehat{\varphi}):=\sigma_{e}(\widehat{\mathbf{u}})-(\zeta \widehat{\theta}) \mathbf{I}+\mathbf{e}^{\top} \nabla \widehat{\varphi}$.

We will make use of Green's third identity to derive the equivalent non-local problem. First, we must represent the solutions of (21) in the form:

$$
\widehat{p}(s)=D(s) \widehat{\phi}-S(s) \widehat{\lambda} \quad \text { in } \Omega^{c},
$$

where the Cauchy data for (21) is given by the densities $\widehat{\phi}:=\hat{p}^{+}(s)$ and $\widehat{\lambda}:=\partial \widehat{p}^{+} / \partial n$, and the simple-layer, $S(s)$, and double-layer, $D(s)$, potentials of the corresponding operator that are defined by

$$
\begin{aligned}
S(s) \widehat{\lambda}(\boldsymbol{x}) & :=\int_{\Gamma} E_{s / c}(\boldsymbol{x}, \boldsymbol{y}) \widehat{\lambda}(\boldsymbol{y}) d \Gamma_{\boldsymbol{y}}, \quad x \in \Omega^{c}, \\
D(s) \widehat{\phi}(\boldsymbol{x}) & :=\int_{\Gamma} \frac{\partial}{\partial n_{y}} E_{s / c}(\boldsymbol{x}, \boldsymbol{y}) \widehat{\phi}(\boldsymbol{y}) d \Gamma_{\boldsymbol{y}}, \quad x \in \Omega^{c} .
\end{aligned}
$$

Here

$$
E_{S / c}(x, y):=\frac{e^{-s|x-y| / c}}{4 \pi|x-y|}
$$


is the fundamental solution of Equation (21). As with their counterpart in the frequencydomain, the Cauchy data $\hat{\lambda}$ and $\hat{\phi}$ satisfy the following integral relations:

$$
\left(\begin{array}{l}
\widehat{\phi} \\
\widehat{\lambda}
\end{array}\right)=\left(\begin{array}{cc}
\frac{1}{2} I+K(s) & -V(s) \\
-W(s) & \left(\frac{1}{2} I-K(s)\right)^{\prime}
\end{array}\right)\left(\begin{array}{c}
\widehat{\phi} \\
\widehat{\lambda}
\end{array}\right) \text { on } \quad \Gamma .
$$

In the preceding relation, $V, K, K^{\prime}$ and $W$ are the four basic boundary integral operators that are defined by:

$$
\left.\begin{array}{rl}
V(s) & :=\{\{\gamma S(s)\}\}=\frac{1}{2}\left(\gamma^{-} S(s)+\gamma^{+} S(s)\right) \\
& =\gamma^{-} S(s)=\gamma^{+} S(s) \\
K(s) & \left.:=\{\{\gamma D(s)\}\}=\frac{1}{2}\left(\gamma^{-} D(s)\right)+\gamma^{+} D(s)\right) \\
K^{\prime}(s) & :=\{\{\gamma S(s)\}\}=\frac{1}{2}\left(\gamma^{-} S(s)+\gamma^{+} S(s)\right) \\
W(s) & :=-\left\{\left\{\partial_{n} D(s)\right\}\right\}=-\frac{1}{2}\left(\partial_{n}^{-} D(s)+\partial_{n}^{+} D(s)\right) \\
& =-\partial_{n}^{-} D(s)=-\partial_{n}^{+} D(s)
\end{array}\right\} \text { on } \quad \Gamma .
$$

In terms of $\hat{\phi}$ and $\widehat{\lambda}$, the two transmission conditions (22) and (23) become:

$$
\begin{aligned}
\sigma(\widehat{\mathbf{u}}, \widehat{\theta}, \widehat{\varphi})^{-} \mathbf{n} & =-\left(\widehat{\phi}(s)+\widehat{p}(s)^{i n c}\right)^{+} \mathbf{n} & & \text { on } \quad \Gamma, \\
-s^{2} \widehat{\mathbf{u}}^{-} \cdot \mathbf{n}+\frac{1}{\rho_{f}}\left(W(s) \widehat{\phi}-\left(\frac{1}{2} I-K(s)\right)^{\prime} \widehat{\lambda}\right) & =\frac{1}{\rho_{f}}\left(\frac{\partial \widehat{p}^{i n c}}{\partial n}\right)^{+} & & \text {on } \quad \Gamma .
\end{aligned}
$$

Using the densities $\widehat{\phi}$ and $\widehat{\lambda}$ as new unknowns, Equation (21) may be eliminated from the problem by using the second equation above together with the boundary integral equation in the first row of (26), namely:

$$
\left(\frac{1}{2} I-K(s)\right) \widehat{\phi}+V(s) \widehat{\lambda}=0 \quad \text { on } \quad \Gamma .
$$

This leads to an integro-differential formulation for the unknowns $(\widehat{\mathbf{u}}, \widehat{\theta}, \widehat{\varphi}, \widehat{\phi}, \widehat{\lambda})$ satisfying the partial differential Equations (18)-(20) in $\Omega$, together with the boundary conditions (23), and (24), and the boundary integral Equations (27) and (28) on $\Gamma$.

Let us first define the space:

$$
H_{*}^{1}(\Omega):=\left\{\varphi \in H^{1}(\Omega) \mid \int_{\Omega} \varphi(x) d x=0\right\},
$$

and restrict our search for the unknown functions $(\widehat{\mathbf{u}}, \widehat{\theta}, \widehat{\varphi})$ to the product space $\mathbf{H}^{1}(\Omega) \times$ $H^{1}(\Omega) \times H_{*}^{1}(\Omega)$. To do so, we multiply Equations (18)-(20) by $(\widehat{\mathbf{v}}, \widehat{\vartheta}, \widehat{\psi}) \in \mathbf{H}^{1}(\Omega) \times$ $H^{1}(\Omega) \times H_{*}^{1}(\Omega)$. Integrating by parts the resuting relations will lead to:

$$
\left.\begin{array}{rl}
a(\widehat{\mathbf{u}}, \widehat{\mathbf{v}} ; s)-\zeta(\widehat{\theta}, \nabla \cdot \widehat{\mathbf{v}})_{\Omega}+(\nabla \widehat{\varphi}, \mathbf{e} \varepsilon(\widehat{\mathbf{v}}))_{\Omega}+\left\langle\widehat{\phi} \mathbf{n}, \widehat{\mathbf{v}}^{-}\right\rangle_{\Gamma} & =-\left\langle\widehat{p}^{i n c+} \mathbf{n}, \widehat{\mathbf{v}}^{-}\right\rangle_{\Gamma} \\
s(\zeta \nabla \cdot \widehat{\mathbf{u}}-\mathbf{p} \cdot \nabla \hat{\varphi}, \widehat{\vartheta})_{\Omega}+\frac{1}{T_{0}} b(\widehat{\theta}, \widehat{\vartheta} ; s)_{\Omega} & =\frac{1}{T_{0}}\left\langle\widehat{f}_{\theta}, \widehat{\vartheta}^{-}\right\rangle_{\Gamma} \\
-(\mathbf{e} \varepsilon(\widehat{\mathbf{u}}), \nabla \widehat{\psi})_{\Omega}-(\widehat{\theta} \mathbf{p}, \nabla \widehat{\psi})_{\Omega}+\epsilon c(\widehat{\varphi}, \widehat{\psi} ; s)_{\Omega}=-\left\langle\widehat{f}_{\mathbf{D}}, \widehat{\psi}^{-}\right\rangle_{\Gamma}
\end{array}\right\}
$$


where $a(\cdot, \cdot ; s), b(\cdot, \cdot ; s)$ and $c(\cdot, \cdot ;, s)$ are sesquilinear forms defined, respectively, by:

$$
\begin{aligned}
a(\widehat{\mathbf{u}}, \widehat{\mathbf{v}} ; s) & :=\left(\sigma_{e}(\widehat{\mathbf{u}}), \varepsilon(\widehat{\mathbf{v}})\right)+s^{2} \rho_{e}(\widehat{\mathbf{u}}, \widehat{\mathbf{v}})_{\Omega} \\
b(\widehat{\theta}, \widehat{\vartheta} ; s)_{\Omega}: & =(\nabla \widehat{\theta}, \nabla \widehat{\vartheta})_{\Omega}+c_{\varepsilon} s(\widehat{\theta}, \widehat{\vartheta})_{\Omega} \\
c(\widehat{\varphi}, \widehat{\psi} ; s)_{\Omega}: & =(\nabla \widehat{\varphi}, \nabla \widehat{\psi})_{\Omega} .
\end{aligned}
$$

Now, let $A_{s}, B_{s}$ and $C_{s}$ be the operators that are defined by the mappings:

$$
\boldsymbol{A}_{s} \widehat{\mathbf{u}}:=a(\widehat{\mathbf{u}}, ; s), \quad B_{s} \widehat{\boldsymbol{\theta}}:=b(\widehat{\theta}, \cdot ; s)_{\Omega}, \quad \text { and } C_{s} \widehat{\varphi}:=c(\widehat{\varphi}, \cdot ; s)_{\Omega},
$$

and consider the function spaces:

$$
\begin{aligned}
X & :=\mathbf{H}^{1}(\Omega) \times H^{1}(\Omega) \times H_{*}^{1}(\Omega) \times H^{1 / 2}(\Gamma) \times H^{-1 / 2}(\Gamma), \\
X^{\prime} & :=\left(\mathbf{H}^{1}(\Omega)\right)^{\prime} \times\left(H^{1}(\Omega)\right)^{\prime} \times\left(H_{*}^{1}(\Omega)\right)^{\prime} \times H^{-1 / 2}(\Gamma) \times H^{1 / 2}(\Gamma), \\
X_{0}^{\prime} & :=\left\{\left(\hat{d}_{1}, \hat{d}_{2}, \hat{d}_{3}, \hat{d}_{4}, \hat{d}_{5}\right) \in X^{\prime} \quad \text { with } \hat{d}_{5}=0\right\} .
\end{aligned}
$$

Subsequently, from Equations (29)-(28), we pose the nonlocal problem as:

The nonlocal boundary problem. For problem data $\left(\widehat{d_{1}}, \widehat{d_{2}}, \widehat{d_{3}}, \widehat{d}_{4}, \widehat{d}_{5}\right) \in X^{\prime}$, given by

$$
\begin{array}{lll}
\widehat{d_{1}}=-\gamma^{-\prime}\left(\gamma^{+} \widehat{p}^{i n c} \mathbf{n}\right), & \widehat{d}_{2}=\gamma^{-\prime}\left(\Theta_{0}^{-1} \widehat{f}_{\theta}\right), \quad \widehat{d_{3}}=-\gamma^{-\prime} \widehat{f}_{\mathbf{D}}, \\
\widehat{d}_{4}=\left(\rho_{f}\right)^{-1} \partial_{n}^{+} \widehat{p}^{i n c}, & \widehat{d_{5}}=0,
\end{array}
$$

find functions $(\widehat{\mathbf{u}}, \widehat{\theta}, \widehat{\varphi}, \widehat{\phi}, \widehat{\lambda}) \in X$ satisfying

$$
\mathbb{A}(s)(\widehat{\mathbf{u}}, \widehat{\theta}, \widehat{\varphi}, \widehat{\phi}, \widehat{\lambda})^{\top}=\left(\widehat{d_{1}}, \widehat{d_{2}}, \widehat{d_{3}}, \widehat{d}_{4}, \widehat{d_{5}}\right)^{\top}
$$

with

$$
\mathbb{A}(s)\left(\begin{array}{c}
\widehat{\mathbf{u}} \\
\widehat{\theta} \\
\widehat{\varphi} \\
\widehat{\phi} \\
\widehat{\lambda}
\end{array}\right):=\left(\begin{array}{ccccc}
\boldsymbol{A}_{s} & -\zeta(\nabla \cdot)^{\prime} & \boldsymbol{\varepsilon}^{\prime} \mathbf{e}^{\top} \nabla & \gamma_{n}^{-{ }^{\prime}} & 0 \\
s \zeta \nabla \cdot & T_{0}^{-1} B_{s} & -s \mathbf{p} \cdot \nabla & 0 & 0 \\
-\nabla^{\prime} \mathbf{e} \boldsymbol{\varepsilon} & -\nabla^{\prime} \mathbf{p} & \epsilon C_{s} & 0 & 0 \\
-s^{2} \gamma_{n}^{-} & 0 & 0 & \rho_{f}^{-1} W(s) & -\rho_{f}^{-1}\left(\frac{1}{2} I-K(s)\right)^{\prime} \\
0 & 0 & 0 & \frac{1}{2} I-K(s) & V(s)
\end{array}\right)\left(\begin{array}{c}
\widehat{\mathbf{u}} \\
\widehat{\theta} \\
\widehat{\phi} \\
\widehat{\phi} \\
\widehat{\lambda}
\end{array}\right) .
$$

In the next section, we will show that this problem is, in fact, well-posed.

\section{Variational Solutions}

We are interested in seeking variational solutions of the nonlocal boundary problem (30) in the transformed domain. To this end, we need some additional preliminary results and definitions. We begin with the norms:

$$
\begin{array}{rlrl}
\|\widehat{\mathbf{u}}\|_{|s|, \Omega}^{2} & :=(\sigma(\widehat{\mathbf{u}}), \widehat{\varepsilon}(\widehat{\hat{\mathbf{u}}}))_{\Omega}+\rho_{e}\||s| \widehat{\mathbf{u}}\|_{\Omega}^{2} & \widehat{\mathbf{u}} \in \mathbf{H}^{1}(\Omega), \\
\|\widehat{\theta}\|_{|s|, \Omega}^{2} & :=\|\nabla \widehat{\theta}\|_{\Omega}^{2}+c_{\mathcal{\varepsilon}}^{-1}\|\sqrt{|s|} \widehat{\theta}\|_{\Omega}^{2} & & \widehat{\theta} \in H^{1}(\Omega), \\
\|\| \widehat{\varphi} \|_{1, \Omega}^{2} & :=\|\nabla \widehat{\varphi}\|_{\Omega}^{2} & & \widehat{\varphi} \in H_{*}^{1}(\Omega), \\
\|\widehat{p}\|_{|s|, \Omega^{c}}^{2} & :=\|\nabla \hat{p}\|_{\Omega^{c}}^{2}+c^{-2}\||s| \widehat{p}\|_{\Omega^{c}}^{2} & & \widehat{p} \in H^{1}\left(\Omega^{c}\right) .
\end{array}
$$

For $\widehat{\varphi} \in H_{*}^{1}(\Omega)$, we see that $\|\nabla \widehat{\varphi}\|_{\Omega}^{2}=0$ if and only if $\widehat{\varphi}=0$. Hence, (32) indeed defines a norm in $H_{*}^{1}(\Omega)$ (see Hsiao and Wendland [Lemma 5.2.5, p.255] [36]). 
We will define $\sigma:=\operatorname{Re} s$ and $\underline{\sigma}:=\min \{1, \sigma\}$. With this notation, it is not hard to verify that:

$$
\underline{\sigma} \leq \min \{1,|s|\}, \quad \text { and } \max \{1,|s|\} \underline{\sigma} \leq|s|, \quad \forall s \in \mathbb{C}_{+}
$$

Using these relations, it is possible to prove the following inequalities relating the energy norms that are defined above

$$
\begin{gathered}
\underline{\sigma}\|\widehat{\mathbf{u}}\|_{1, \Omega} \leq\|\widehat{\mathbf{u}}\|_{|s|, \Omega} \leq \frac{|s|}{\underline{\sigma}}\|\widehat{\mathbf{u}}\|_{1, \Omega}, \\
\sqrt{\underline{\sigma}}\|\widehat{\theta}\|_{1, \Omega} \leq\|\widehat{\theta}\|\left\|_{|s|, \Omega_{+}} \leq \sqrt{\frac{|s|}{\underline{\sigma}}}\right\| \widehat{\theta} \|_{1, \Omega}, \\
\underline{\sigma}\|\widehat{p}\|_{1, \Omega_{+}} \leq\|\widehat{p}\|_{|s|, \Omega_{+}} \leq \frac{|s|}{\underline{\sigma}}\|\widehat{p}\|_{1, \Omega_{+}} .
\end{gathered}
$$

These relations will be used heavily when estimating the norms of the solutions in terms of the Laplace parameter $s$ and its real part $\sigma$. The norms $\|\cdot\| \|_{1, \Omega}$ and $\|\cdot\|_{1, \Omega^{c}}$ are, respectively, equivalent to $\|\cdot\|_{H^{1}(\Omega)}$ and $\|\cdot\|_{H^{1}\left(\Omega^{c}\right)}$. An application of Korn's second inequality [38] shows that, for a vector-valued function $\widehat{\mathbf{u}}$, the energy norm $\|\cdot\|_{1, \Omega}$ is also equivalent to the standard $\mathbf{H}^{1}(\Omega)$ norm. Now, given a vector of solutions $(\widehat{\mathbf{u}}, \widehat{\theta}, \widehat{\varphi}, \widehat{\phi}, \widehat{\lambda})$ to (30), by defining

$$
\widehat{p}(s)=D(s) \widehat{\phi}-S(s) \widehat{\lambda} \quad \text { in } \quad \mathbb{R}^{3} \backslash \Gamma,
$$

then $\hat{p} \in H^{1}\left(\mathbb{R}^{3} \backslash \Gamma\right)$ is the unique solution of the transmission problem:

$$
\begin{aligned}
-\Delta \widehat{p}+\frac{s^{2}}{c^{2}} \widehat{p}(s) & =0 & & \text { in } \quad \mathbb{R}^{3} \backslash \Gamma, \\
\llbracket \gamma \hat{p} \rrbracket & =\widehat{\phi} \in H^{1 / 2}(\Gamma) & & \text { on } \quad \Gamma, \\
\llbracket \partial_{n} \hat{p} \rrbracket & =\widehat{\lambda} \in H^{-1 / 2}(\Gamma) & & \text { on } \quad \Gamma,
\end{aligned}
$$

where the symbol $\llbracket \cdot \rrbracket$ denotes the "jump" relations of a function across $\Gamma$. More specifically, we have

$$
\llbracket \gamma \hat{p} \rrbracket:=\left(\hat{p}^{+}-\widehat{p}^{-}\right), \quad \text { and } \quad \llbracket \partial_{n} \hat{p} \rrbracket:=\left(\partial_{n}^{+} \hat{p}-\partial_{n}^{-} \hat{p}\right) .
$$

We remark that, in the present case, no radiation condition is needed to ensure uniquness because of Huygen's principle. In terms of the jumps of $\hat{p}$, the last two equations of (30) are equivalent to

$$
\begin{aligned}
-s^{2} \gamma_{n}^{-} \widehat{\mathbf{u}}-\frac{1}{\rho_{f}} \partial_{n}^{+} \widehat{p} & =\frac{1}{\rho_{f}} \widehat{d}_{4} & \text { on } \quad \Gamma \\
-\gamma^{-} \hat{p} & =\widehat{d}_{5} & \text { on } \quad \Gamma
\end{aligned}
$$

Because $\widehat{d_{5}}=0$, we conclude that $\widehat{p}$ satisfies the homogeneous Dirichlet problem for (36) in $\Omega$ and, by uniqueness, it must follow that $\hat{p} \equiv 0$ in $\bar{\Omega}$. As a consequence, we have the following relations between the unknown densities and Cauchy data:

$$
\llbracket \gamma \hat{p} \rrbracket=\gamma^{+} \widehat{p}=\widehat{\phi} \quad \text { and } \quad \llbracket \partial_{n} \hat{p} \rrbracket=\partial_{n}^{+} \hat{p}=\widehat{\lambda}
$$

On the other hand, the transmission condition (37) is closely related to the variational equation of Equation (36)

$$
\begin{aligned}
-\left\langle\partial_{n}^{+} \hat{p}, \overline{\gamma^{+} \hat{q}}\right\rangle_{\Gamma} & =\int_{\Omega^{c}}\left(\nabla \hat{p} \cdot \overline{\nabla \widehat{q}}+(s / c)^{2} \hat{p} \overline{\hat{q}}\right) d x \\
& =: d_{\Omega^{c}}(\widehat{p}, \widehat{q} ; s) \\
& =:\left(D_{s} \hat{p}, \widehat{q}\right)_{\Omega^{c}}
\end{aligned}
$$


where the domain of integration for the sesquilinear form $d_{\Omega^{c}}(\widehat{p}, \widehat{q} ; s)$ and the associated operator $D_{s}$, has been explicitly indicated in the definition. Now, using (37), we arrive at

$$
-s^{2}\left\langle\gamma^{-\widehat{\mathbf{u}}}, \overline{\gamma^{+} \widehat{q}} \mathbf{n}\right\rangle_{\Gamma}+\frac{1}{\rho_{f}}\left(D_{s} \widehat{p}, \overline{\hat{q}}\right)_{\Omega^{c}}=\left\langle\widehat{d}_{4}, \overline{\gamma^{+} \widehat{q}}\right\rangle_{\Gamma} .
$$

Combining the above equality with the weak formulations of the first three Equations in (30), we can formulate an equivalent variational problem. We will first introduce the space $\mathbb{H}:=\mathbf{H}^{1}(\Omega) \times H^{1}(\Omega) \times H_{*}^{1}(\Omega) \times H^{1}\left(\Omega^{c}\right)$ and then endow it with the norm:

$$
\|(\widehat{\mathbf{u}}, \widehat{\theta}, \widehat{\varphi}, \widehat{p})\|_{\mathbb{H}}:=\left(\|\widehat{\mathbf{u}}\|_{1, \Omega}^{2}+\|\widehat{\theta}\|_{1, \Omega}^{2}+\|\widehat{\varphi}\|_{\Omega}^{2}+\|\widehat{p}\|_{1, \Omega^{c}}^{2}\right)^{1 / 2} \text {. }
$$

The variational problem. Find $(\widehat{\mathbf{u}}, \widehat{\theta}, \widehat{\varphi}, \widehat{p}) \in \mathbb{H}$ satisfying

$$
\mathcal{A}((\widehat{\mathbf{u}}, \widehat{\theta}, \widehat{\varphi}, \widehat{p}),(\widehat{\mathbf{v}}, \widehat{\vartheta}, \widehat{\psi}, \widehat{q}) ; s)=\ell_{d}((\widehat{\mathbf{v}}, \widehat{\vartheta}, \widehat{\psi}, \widehat{q})), \quad \forall(\widehat{\mathbf{v}}, \widehat{\vartheta}, \widehat{\psi}, \widehat{q}) \in \mathbb{H}
$$

where the sesquilinear form on the left hand side of the equation is defined by:

$$
\begin{aligned}
\mathcal{A}((\widehat{\mathbf{u}}, \widehat{\theta}, \widehat{\varphi}, \widehat{p}),(\widehat{\mathbf{v}}, \widehat{\vartheta}, \widehat{\psi}, \widehat{q}) ; s):= & \left(A_{s} \widehat{\mathbf{u}}, \overline{\mathbf{v}}\right)_{\Omega}-\zeta(\widehat{\theta}, \nabla \cdot \overline{\mathbf{v}})_{\Omega}+(\nabla \widehat{\varphi}, \mathbf{e} \varepsilon(\overline{\mathbf{v}}))_{\Omega}+\left\langle\gamma^{+} \widehat{p} \mathbf{n}, \gamma^{-\overline{\mathbf{v}}\rangle_{\Gamma}}\right. \\
& +s \zeta(\nabla \cdot \widehat{\mathbf{u}}, \overline{\widehat{\vartheta}})_{\Omega}+T_{0}^{-1}\left(B_{s} \widehat{\theta}, \overline{\hat{\vartheta}}\right)_{\Omega}-s(\mathbf{p} \cdot \nabla \widehat{\varphi}, \bar{\vartheta})_{\Omega} \\
& -(\mathbf{e} \varepsilon(\widehat{\mathbf{u}}), \nabla \bar{\psi})_{\Omega}-(\mathbf{p} \widehat{\theta}, \nabla \bar{\psi})_{\Omega}+\epsilon\left(C_{s} \widehat{\varphi}, \bar{\psi}\right)_{\Omega} \\
& -s^{2}\left\langle\gamma^{-} \widehat{\mathbf{u}}, \bar{\gamma} \bar{\gamma} \hat{q}\right\rangle_{\Gamma}+\frac{1}{\rho_{f}}\left(D_{s} \widehat{p}, \overline{\hat{q}}\right)_{\Omega^{c}}
\end{aligned}
$$

for $(\widehat{\mathbf{v}}, \widehat{\vartheta}, \widehat{\psi}, \widehat{q}) \in \mathbb{H}$. The bounded linear functional on the right hand side is defined by

$$
\ell_{d}((\widehat{\mathbf{v}}, \widehat{\vartheta}, \widehat{\psi}, \widehat{q})):=\left(\widehat{d_{1}}, \overline{\mathbf{v}}\right)_{\Omega}+\left(\widehat{d_{2}}, \overline{\widehat{\vartheta}}\right)_{\Omega}+\left(\widehat{d_{3}}, \overline{\widehat{\psi}}\right)_{\Omega}+\left\langle\widehat{d_{4}}, \overline{\gamma^{+} \widehat{q}}\right\rangle_{\Gamma},
$$

for all tests $(\widehat{\mathbf{v}}, \widehat{\vartheta}, \widehat{\psi}, \widehat{q}) \in \mathbb{H}$. By construction, this variational problem is equivalent to the transmission problem (18) through (24) which in turn is equivalent to (30). Consequently, it suffices to show the existence of a solution of (39) in order to guarantee that (30) is indeed solvable. We now present the following basic existence and uniqueness results.

Theorem 1. Under the assumption of the constant pyroelectric moduli vector vector $\mathbf{p}$ satisfying the constraint

$$
\|\mathbf{p}\|_{\mathbb{R}^{3}}<\min \left\{\epsilon, \frac{C_{\varepsilon}}{T_{0}}\right\},
$$

the variational problem (39) has a unique solution $(\widehat{\mathbf{u}}, \widehat{\theta}, \widehat{\varphi}, \widehat{p}) \in \mathbb{H}$. Moreover, the following estimate holds:

$$
\|(\widehat{\mathbf{u}}, \widehat{\theta}, \widehat{\varphi}, \widehat{p})\|_{\mathbb{H}} \leq c_{0} \frac{|s|^{3}}{\sigma \underline{\sigma}^{6}}\left\|\left(\widehat{d_{1}}, \widehat{d_{2}}, \widehat{d_{3}}, \widehat{d_{4}}\right)\right\|_{\mathbb{H}^{\prime}}
$$

Here, and in the sequel, $c_{0}>0$ will denote a constant that may only depend on $\rho_{f}, T_{0}, c_{\varepsilon}, \epsilon, \mathbf{p}$.

Proof. Let $\mathcal{A}((\widehat{\mathbf{u}}, \widehat{\theta}, \widehat{\varphi}, \widehat{p}),(\widehat{\mathbf{v}}, \widehat{\vartheta}, \widehat{\psi}, \widehat{q}) ; s)$ be the sesquilinear form that is defined by the variational Equation (39). We first show that $\mathcal{A}$ is continuous. It is easy to verify that

$$
\begin{aligned}
\left|\left(A_{s} \widehat{\mathbf{u}}, \overline{\mathbf{v}}\right)_{\Omega}+T_{0}^{-1}\left(B_{s} \widehat{\theta}, \overline{\hat{\vartheta}}\right)_{\Omega}+\epsilon\left(C_{s} \widehat{\varphi}, \overline{\hat{\psi}}\right)_{\Omega}+\frac{1}{\rho_{f}}\left(D_{s} \widehat{p}, \overline{\hat{q}}\right)_{\Omega^{c}}\right| \leq \\
m_{1}\left(\frac{|s|}{\underline{\sigma}}\right)^{2}\|(\mathbf{u}, \theta, \varphi, p)\|_{\mathbb{H}}\|(\widehat{\mathbf{v}}, \widehat{\vartheta}, \widehat{\psi}, \widehat{q})\|_{\mathbb{H}} .
\end{aligned}
$$


The remaining terms in $\mathcal{A}((\widehat{\mathbf{u}}, \widehat{\theta}, \widehat{\varphi}, \widehat{p}),(v, \vartheta, \psi, q))$ can be easily bounded using the Cauchy-Schwartz inequality, Poicaré's inequality in $H_{*}^{1}(\Omega)$, the trace theorem, and the estimate

$$
\left|(\nabla \varphi, \mathbf{e} \varepsilon(\mathbf{v}))_{\Omega}\right| \leq \mathrm{e}_{\max }\|\nabla \varphi\|_{\Omega}\|\nabla \cdot \mathbf{v}\|_{\Omega} .
$$

This leads to the continuity estimate

$$
\mathcal{A}((\widehat{\mathbf{u}}, \widehat{\theta}, \widehat{\varphi}, \widehat{p}),(\widehat{\mathbf{v}}, \widehat{\vartheta}, \widehat{\psi}, \widehat{q}) ; s) \leq\left(m_{1}+m_{2}\right)\left(\frac{|s|}{\underline{\sigma}}\right)^{2}\|(\mathbf{u}, \theta, \varphi, p)\|_{\mathbb{H}}\|(\widehat{\mathbf{v}}, \widehat{\vartheta}, \widehat{\psi}, \widehat{q})\|_{\mathbb{H}} .
$$

Here, $m_{1}$ and $m_{2}$ are constants only depending upon the physical parameters $\zeta, \Theta_{0}, \mathbf{p}, \epsilon$, and $\mathrm{e}_{\max }=\max \left\{\mathrm{e}_{i j k}, i, j, k=1 \cdots 3\right\}$. We now introduce the scaling factor:

$$
Z(s):=\left(\begin{array}{cccc}
\bar{s} & 0 & 0 & 0 \\
0 & 1 & 0 & 0 \\
0 & 0 & s & 0 \\
0 & 0 & 0 & \bar{s} /|s|^{2}
\end{array}\right),
$$

and note that, for $(\widehat{\mathbf{u}}, \widehat{\theta}, \widehat{\varphi}, \widehat{p}) \in \mathbb{H}$, we have

$$
\begin{aligned}
& \operatorname{Re}\left(\bar{s}\left(-\zeta(\widehat{\theta}, \nabla \cdot \overline{\widehat{\mathbf{u}}})_{\Omega}+\left(\mathbf{e}^{\top} \nabla \widehat{\varphi}, \varepsilon(\widehat{\widehat{\mathbf{u}}})\right)_{\Omega}+\left\langle\gamma^{+} \widehat{p} \mathbf{n}, \gamma^{-} \overline{\widehat{\mathbf{u}}}\right\rangle_{\Gamma}\right)\right. \\
& \left.\quad+s\left(\zeta(\nabla \cdot \widehat{\mathbf{u}}, \overline{\widehat{\theta}})_{\Omega}-(\mathbf{e} \varepsilon(\widehat{\mathbf{u}}), \nabla \overline{\hat{\varphi}})_{\Omega}\right)-\left(\bar{s} /|s|^{2}\right) s^{2}\left\langle\gamma^{-} \widehat{\mathbf{u}}, \overline{\gamma^{+} \widehat{p}} \mathbf{n}\right\rangle_{\Gamma}\right)=0 .
\end{aligned}
$$

Therefore, it follows that:

$$
\begin{aligned}
& \operatorname{Re}(Z(s) \mathcal{A}((\widehat{\mathbf{u}}, \widehat{\theta}, \widehat{\varphi}, \widehat{p}),(\widehat{\mathbf{u}}, \widehat{\theta}, \widehat{\varphi}, \widehat{p}) ; s))=\operatorname{Re}\left(\bar{s}\left(\boldsymbol{A}_{s} \widehat{\mathbf{u}}, \widehat{\widehat{\mathbf{u}}}\right)_{\Omega}+T_{0}^{-1}\left(B_{s} \widehat{\theta}, \widehat{\widehat{\theta}}\right)_{\Omega}\right. \\
&-s\left((\mathbf{p} \cdot \nabla \widehat{\varphi}, \overline{\hat{\theta}})_{\Omega}-(\mathbf{p} \widehat{\theta}, \nabla \overline{\hat{\varphi}})_{\Omega}+\epsilon\left(C_{s} \widehat{\varphi}, \overline{\hat{\varphi}}\right)_{\Omega}\right) \\
&\left.+\left(\bar{s} /|s|^{2}\right) \rho_{f}^{-1}\left(D_{s} \widehat{p}, \overline{\hat{p}}\right)_{\Omega^{c}}\right) .
\end{aligned}
$$

By setting to zero some of the entries of $(\widehat{\mathbf{u}}, \widehat{\theta}, \widehat{\varphi}, \widehat{p})$ in the right hand side of $(42)$, it is possible to derive the following:

$$
\begin{aligned}
\left.\operatorname{Re}\left(\bar{s}\left(\boldsymbol{A}_{s} \widehat{\mathbf{u}}, \widehat{\mathbf{u}}\right)_{\Omega}\right)\right) & =\sigma\|\widehat{\mathbf{u}}\|_{|s|, \Omega}^{2} \\
\operatorname{Re}\left(T_{0}^{-1}\left(B_{s} \widehat{\theta}, \widehat{\widehat{\theta}}\right)_{\Omega}\right) & =T_{0}^{-1}\left(\|\nabla \widehat{\theta}\|_{\Omega}^{2}+c_{\varepsilon} \sigma\|\widehat{\theta}\|^{2}\right) \\
\operatorname{Re}\left(-s\left((\mathbf{p} \cdot \nabla \widehat{\varphi}, \widehat{\hat{\theta}})_{\Omega}+(\mathbf{p} \widehat{\theta}, \nabla \overline{\hat{\varphi}})_{\Omega}\right)\right) & \geq-\sigma\|\mathbf{p}\|_{\mathbb{R}^{3}}\left(\|\nabla \varphi\|_{\Omega}+\|\theta\|_{\Omega}\right) \\
\operatorname{Re}\left(s \epsilon\left(C_{s} \widehat{\varphi}, \overline{\hat{\varphi}}\right)_{\Omega}\right) & =\sigma \epsilon\|\nabla \varphi\|_{\Omega}^{2} \\
\operatorname{Re}\left(\left(\bar{s} /|s|^{2}\right) \rho_{f}^{-1}\left(D_{s} \widehat{p}, \overline{\hat{p}}\right)_{\Omega^{c}}\right) & =\left(\sigma /|s|^{2}\right) \rho_{f}^{-1}\|p\|_{|s|, \Omega^{c}}^{2}
\end{aligned}
$$

From Equatons (43) and (42), it follows that:

$$
\operatorname{Re}(Z(s) \mathcal{A}((\widehat{\mathbf{u}}, \widehat{\theta}, \widehat{\varphi}, \widehat{p}),(\widehat{\mathbf{u}}, \widehat{\theta}, \widehat{\varphi}, \widehat{p}) ; s)) \geq \frac{\sigma \sigma^{2}}{|s|^{2}}\left(\|\widehat{\mathbf{u}}\|\left\|_{|s|, \Omega}^{2}+c_{1}\right\| \widehat{\theta}\left\|_{|s|, \Omega}^{2}+c_{2}\right\| \widehat{\varphi}\left\|_{\Omega}^{2}+\right\| \hat{p} \|_{|s|, \Omega}^{2}\right),
$$

where $c_{1}=c_{\varepsilon}^{-1}\left(c_{\varepsilon} \Theta_{0}^{-1}-\|\widehat{\mathbf{p}}\|_{\mathbb{R}^{3}}\right)>0$ and $c_{2}=\left(\epsilon-\|\mathbf{p}\|_{\mathbb{R}^{3}}\right)>0$. Alternatively, in view of (33)-(35), we have

$$
|\mathcal{A}((\widehat{\mathbf{u}}, \widehat{\theta}, \widehat{\varphi}, \widehat{p}),(\widehat{\mathbf{u}}, \widehat{\theta}, \widehat{\varphi}, \widehat{p}) ; s)| \geq \alpha_{0} \frac{\sigma \underline{\sigma}^{6}}{|s|^{3}}\|(\widehat{\mathbf{u}}, \widehat{\theta}, \widehat{\varphi}, \widehat{p})\|_{\mathbb{H}}^{2}
$$

where $\alpha_{0}>0$ is a constant independent of $\sigma$, and $|s|$. Hence, by the Lax-Milgram lemma, there exists a unique solution of the variational problem (39). 
Having shown that the problem is uniquely solvable, the stability estimate (40) can be derived from (44) and (39), as we show next:

$$
\begin{aligned}
\frac{\sigma \underline{\sigma}^{2}}{|s|^{2}}\left(\|\widehat{\mathbf{u}}\|_{|s|, \Omega}^{2}+c_{1}\|\widehat{\theta}\|_{|s|, \Omega}^{2}+\right. & c_{2} \\
& \left.\|\widehat{\varphi}\|_{\Omega}^{2}+\|\hat{p}\|_{|s|, \Omega^{c}}^{2}\right) \leq \operatorname{Re}(Z(s) \mathcal{A}((\widehat{\mathbf{u}}, \widehat{\theta}, \widehat{\varphi}, \widehat{p}),(\widehat{\mathbf{u}}, \widehat{\theta}, \widehat{\varphi}, \widehat{p}) ; s)) \\
& \leq\left.\left|\bar{s}\left(\widehat{d_{1}}, \widehat{\mathbf{u}}\right)_{\Omega}+\left(\widehat{d_{2}}, \widehat{\theta}\right)_{\Omega}+s\left(\widehat{d_{3}}, \widehat{\varphi}\right)_{\Omega}+\bar{s} /\right| s\right|^{2}\left\langle\widehat{d_{4}}, \gamma^{+} \widehat{p}\right\rangle_{\Gamma} \mid \\
& \leq \frac{|s|}{\underline{\sigma}^{2}}\left(\left|\left(\widehat{d_{1}}, \widehat{\mathbf{u}}\right)_{\Omega}\right|+\left|\left(\widehat{d_{2}}, \widehat{\theta}\right)_{\Omega}\right|+\left|\left(\widehat{d_{3}}, \varphi\right)_{\Omega}\right|+\left|\left\langle\widehat{d_{4}}, \gamma^{+} \widehat{p}\right\rangle_{\Gamma}\right|\right) .
\end{aligned}
$$

Consequently, using the first inequality of the equivalences (33) through (35), we have the estimate:

$$
\left(\|\widehat{\mathbf{u}}\|_{|s|, \Omega}^{2}+\|\widehat{\theta}\|_{|s|, \Omega}^{2}+\|\widehat{\varphi}\|_{\Omega}^{2}+\|\widehat{p}\|_{|s|, \Omega^{c}}^{2}\right)^{1 / 2} \leq c_{0} \frac{|s|^{3}}{\sigma \underline{\sigma}^{5}}\left\|\left(\widehat{d_{1}}, \widehat{d_{2}}, \widehat{d_{3}}, \widehat{d_{4}}, 0\right)\right\|_{X^{\prime}}
$$

Here, $c_{0}$ is a constant only depending on the physical parameters $\rho_{f}, T_{0}, c_{\varepsilon}, \epsilon, \mathbf{p}$. The desired estimate (40) can then be easily derived by simplifying the right hand side of the expression above and applying (33) through (35) to the term on left hand side.

The estimate (45) will lead us to verify the invertibility of the operator matrix $\mathbb{A}(s)$ that is defined in (31), as we now show.

Theorem 2. The operator $\mathbb{A}(s): X \rightarrow X_{0}^{\prime}$, as defined in (31) is invertible. Moreover, we have the estimate:

$$
\left\|\left.\mathbb{A}^{-1}(s)\right|_{X_{0}^{\prime}}\right\|_{X^{\prime}, X} \leq c_{0} \frac{|s|^{3+1 / 2}}{\sigma \underline{\sigma}^{6+1 / 2}}
$$

Proof. From Equation (38), we see that

$$
\llbracket \gamma \hat{p} \rrbracket=\gamma^{+} \hat{p}=\widehat{\phi} \quad \text { and } \quad \llbracket \partial_{n} \hat{p} \rrbracket=\partial_{n}^{+} \hat{p}=\widehat{\lambda} .
$$

From which it can be shown that (see, e.g., [39]):

$$
\|\widehat{\phi}\|_{H^{1 / 2}(\Gamma)}^{2}=\left\|\gamma^{+} \hat{p}\right\|_{H^{1 / 2}(\Gamma)}^{2} \leq c_{1}\|\hat{p}\|_{1, \Omega^{c}}^{2} \leq c_{1} \frac{1}{{\underline{\sigma^{2}}}^{2}}\|\widehat{p}\|_{|\hat{s}|, \Omega^{c}}^{2}
$$

Similarly, we have

$$
\left|\left\langle\widehat{\lambda}, \widehat{q}^{+}\right\rangle\right|=\left|\left\langle\partial_{n}^{+} \widehat{p}, \widehat{q}^{+}\right\rangle\right|=\left|a_{s, \Omega^{c}}(\widehat{p}, \widehat{q})\right| \leq\|\widehat{p}\|_{|s|, \Omega^{c}}\|\widehat{q}\|_{|s|, \Omega^{c}} \leq c_{2} \sqrt{|s| / \underline{\sigma}}\|\widehat{p}\|_{|s|, \Omega^{c}}\left\|\widehat{q}^{+}\right\|_{H^{1 / 2}(\Gamma)}
$$

which implies

$$
\|\widehat{\lambda}\|_{H^{-1 / 2}(\Gamma)}=\sup _{0 \neq q^{+} \in H^{-1 / 2}(\Gamma)} \frac{\left|\left\langle\partial_{n}^{+} \hat{p}, \widehat{q}^{+}\right\rangle_{\Gamma}\right|}{\left\|\widehat{q}^{+}\right\|_{H^{1 / 2}(\Gamma)}} \leq c_{2} \sqrt{|s| / \underline{\sigma}}\|\hat{p}\|_{|s|, \Omega^{c}}
$$

Above, Bamberger and Ha-Duong's optimal lifting [33,34] has been used to bound the norm $\|\widehat{q}\|_{|s|, \Omega^{c}}$ by $\left\|\widehat{q}^{+}\right\|_{H^{1 / 2}(\Gamma)}$ in (48). Subsequetly, (47) and (48) yield the estimates

$$
\frac{1}{2}\left(\frac{1}{c_{1}} \underline{\sigma}^{2}\|\widehat{\phi}\|_{H^{1 / 2}(\Gamma)}^{2}+\frac{\underline{\sigma}}{c_{2}^{2}|s|}\|\widehat{\lambda}\|_{H^{-1 / 2}(\Gamma)}^{2}\right) \leq\|\widehat{p}\|_{|s|, \Omega_{+}}^{2} .
$$


As a consequence of (45), it follows from (49) that

$$
\begin{aligned}
\left(\underline{\sigma}^{2}\|\widehat{\mathbf{u}}\|_{1, \Omega}^{2}+\underline{\sigma}\|\widehat{\theta}\|_{1, \Omega}^{2}+\|\widehat{\varphi}\|_{\Omega}^{2}\right. & \left.+\frac{1}{2}\left(\frac{\sigma^{2}}{c_{1}}\|\widehat{\phi}\|_{H^{1 / 2}(\Gamma)}^{2}+\frac{\underline{\sigma}}{c_{2}^{2}|s|}\|\widehat{\lambda}\|_{H^{-1 / 2}(\Gamma)}^{2}\right)\right)^{1 / 2} \\
& \leq c_{0} \frac{|s|^{3}}{\sigma \underline{\sigma}^{5}}\left\|\left(\widehat{d_{1}}, \widehat{d_{2}}, \widehat{d_{3}}, \widehat{d_{4}}, \widehat{d_{5}}\right)\right\|_{X_{0}^{\prime}}
\end{aligned}
$$

which implies

$$
\begin{aligned}
\left(\|\widehat{\mathbf{u}}\|_{1, \Omega_{-}}^{2}+\|\widehat{\theta}\|_{1, \Omega_{-}}^{2}+\|\widehat{\varphi}\|_{H_{*}^{1}(\Omega)}^{2}+\|\widehat{\phi}\|_{H^{1 / 2}(\Gamma)}^{2}+\|\widehat{\lambda}\|_{H^{-1 / 2}(\Gamma)}^{2}\right)^{1 / 2} & \\
& \leq c_{0} \frac{|s|^{3+1 / 2}}{\sigma \underline{\sigma}^{6+1 / 2}}\left\|\left(\widehat{d_{1}}, \widehat{d_{2}}, \widehat{d_{3}}, \widehat{d}_{4}, \widehat{d}_{5}\right)\right\|_{X_{0}^{\prime}} .
\end{aligned}
$$

\section{Results in the Time Domain}

Having established the properties of the operators and solutions to our problem in the Laplace domain, we can now return to the time domain and establish analogue results. Let us first define a class of admissible symbols in order to state the result that will allow us to transfer our previous analysis in the Laplace domain back in to the time domain, following [18].

The following definition, and the proposition following immediately after it (an improved version of [Proposition 3.2.2] [37,40]) will be used to transform the Laplace-domain bounds into time-domain statements.

A class of admissible symbols: let $\mathbb{X}$ and $\mathbb{Y}$ be Banach spaces and $\mathcal{B}(\mathbb{X}, \mathbb{Y})$ be the set of bounded linear operators from $\mathbb{X}$ to $\mathbb{Y}$. An operator-valued analytic function $A: \mathbb{C}_{+} \rightarrow$ $\mathcal{B}(\mathbb{X}, \mathbb{Y})$ is said to belong to the class $\mathcal{A}(\mu, \mathcal{B}(\mathbb{X}, \mathbb{Y}))$, if there exists a real number $\mu$, such that

$$
\|A(s)\|_{\mathbb{X}, \mathbb{Y}} \leq C_{A}(\operatorname{Re}(s))|s|^{\mu} \quad \text { for } \quad s \in \mathbb{C}_{+},
$$

where the function $C_{A}:(0, \infty) \rightarrow(0, \infty)$ is non-increasing and satisfies

$$
C_{A}(\sigma) \leq \frac{c}{\sigma^{m}}, \quad \forall \quad \sigma \in(0,1]
$$

for some $m \geq 0$ and $c$ independent of $\sigma$.

Proposition 1. ([40]) Let $A=\mathcal{L}\{a\} \in \mathcal{A}(k+\alpha, \mathcal{B}(\mathbb{X}, \mathbb{Y}))$ with $\alpha \in[0,1)$ and $k$ a non-negative integer. If $g \in \mathcal{C}^{k+1}(\mathbb{R}, \mathbb{X})$ is causal and its derivative $g^{(k+2)}$ is integrable, then $a * g \in \mathcal{C}(\mathbb{R}, \mathbb{Y})$ is causal and

$$
\|(a * g)(t)\|_{\mathbb{Y}} \leq 2^{\alpha} C_{\epsilon}(t) C_{A}\left(t^{-1}\right) \int_{0}^{1}\left\|\left(\mathcal{P}_{2} g^{(k)}\right)(\tau)\right\|_{\mathbb{X}} d \tau
$$

where

$$
C_{\epsilon}(t):=\frac{1}{2 \sqrt{\pi}} \frac{\Gamma(\epsilon / 2)}{\Gamma((\epsilon+1) / 2)} \frac{t^{\epsilon}}{(1+t)^{\epsilon}}, \quad(\epsilon:=1-\alpha \text { and } \mu=k+\alpha)
$$

and

$$
\left(\mathcal{P}_{2} g\right)(t)=g+2 \dot{g}+\ddot{g} .
$$

The results that are proven in Section 4-specifically the bounds obtained in terms of the Laplace parameter $s$ and its real part $\sigma$-will now allow us to show that the operators involved belong precisely to one such class of symbols. 
We begin with the results in Theorem 1 and, from (40), we may write

$$
\begin{gathered}
(\widehat{\mathbf{u}}, \widehat{\theta}, \widehat{\varphi}, \widehat{p})^{\top}=A(s)\left(\widehat{d_{1}}, \widehat{d_{2}}, \widehat{d_{3}}, \widehat{d_{4}}, 0\right)^{\top}, \\
\left\|\left.A(s)\right|_{X_{0}^{\prime}}\right\|_{X^{\prime}, \mathbb{H}} \leq C_{A} \frac{|s|^{3}}{\sigma \underline{\sigma}^{6}} .
\end{gathered}
$$

Hence, $A(s) \in \mathcal{A}\left(3, \mathcal{B}\left(X_{0}^{\prime}, \mathbb{H}\right)\right)$, and

$$
\begin{aligned}
(\mathbf{u}, \theta, \varphi, p)^{\top} & =\mathcal{L}^{-1}\left\{A(s)\left(\widehat{d_{1}}, \widehat{d_{2}}, \widehat{d_{3}}, \widehat{d_{4}}, 0\right)^{\top}\right\} \\
& =\mathcal{L}^{-1}\{A(s)\} * \mathcal{L}^{-1}\left\{\left(\widehat{d_{1}}, \widehat{d_{2}}, \widehat{d_{3}}, \widehat{d}_{4}, 0\right)^{\top}\right\} \\
& =\left(\mathcal{L}^{-1}\{A\} * \mathbf{D}\right)(t) \\
& =:(a * g)(t) \quad \text { according to Proposition } 1 .
\end{aligned}
$$

From the estimate of $A(s)$ in Equation (50), we have

$$
\mu=k+\alpha=3 \text { implies } k=3, \alpha=0 \text { and } \varepsilon=1-\alpha=1 .
$$

Thus, we have established the following theorem.

Theorem 3. Let $\mathbb{H}:=\mathbf{H}^{1}(\Omega) \times H^{1}(\Omega) \times H_{*}^{1}(\Omega) \times H^{1}\left(\Omega^{c}\right)$. If

$$
\mathbf{D}(t):=\mathcal{L}^{-1}\left\{\left(\widehat{d_{1}}, \widehat{d_{2}}, \widehat{d_{3}}, \widehat{d}_{4}, 0\right)^{\top}\right\} \in \mathcal{C}^{4}\left(\mathbb{R}, X_{0}^{\prime}\right)
$$

is causal and its derivative $\mathbf{D}^{(5)}$ is integrable, then $(\mathbf{u}, \theta, \varphi, p)^{\top} \in \mathcal{C}(\mathbb{R}, \mathbb{H})^{\top}$ is causal and

$$
\left\|(\mathbf{u}, \theta, \varphi, p)^{\top}(t)\right\|_{\mathbb{H}} \leq c_{0} \frac{t^{2}}{1+t} \max \left\{1, t^{6}\right\} \int_{0}^{t}\left\|\left(\mathcal{P}_{2} \mathbf{D}^{(3)}\right)(\tau)\right\| d \tau
$$

for some constant $c_{0}>0$, where $\left(\mathcal{P}_{2} \mathbf{D}\right)(t)=\mathbf{D}+2 \dot{\mathbf{D}}+\ddot{\mathbf{D}}$.

Similarly, from Theorem 2, we have

$$
(\widehat{\mathbf{u}}, \widehat{\theta}, \widehat{\varphi}, \widehat{\phi}, \widehat{\lambda})^{\top}=\mathbb{A}^{-1}(s)\left(\widehat{d_{1}}, \widehat{d}_{2}, \widehat{d}_{3}, \widehat{d}_{4}, \widehat{d}_{5}\right)^{\top},
$$

from which, while using (46), we infer that

$$
\left\|\left.\mathbb{A}^{-1}(s)\right|_{X_{0}^{\prime}}\right\|_{X^{\prime}, \mathbb{H}} \leq c_{0} \frac{|s|^{3+1 / 2}}{\sigma \underline{\sigma}^{6+1 / 2}}
$$

hence, $\mathbb{A}^{-1}(s) \in \mathcal{A}\left(3 \frac{1}{2}, \mathcal{B}\left(X_{0}^{\prime}, \mathbb{H}\right)\right)$. Applying Proposition 1 with

$$
\mu=(k+\alpha)=3 \frac{1}{2}, k=3, \alpha=1 / 2, \varepsilon=1-\alpha=1 / 2,
$$

then yields the following theorem.

Theorem 4. Let $X:=\mathbf{H}^{1}(\Omega) \times H^{1}(\Omega) \times H_{*}^{1}(\Omega) \times H^{1 / 2}(\Gamma) \times H^{-1 / 2}(\Gamma)$. If

$$
\mathbf{D}(t):=\mathcal{L}^{-1}\left\{\left(\widehat{d_{1}}, \widehat{d_{2}}, \widehat{d_{3}}, \widehat{d_{4}}, \widehat{d_{5}}\right)^{\top}\right\}(t) \in \mathcal{C}^{(4)}\left(\mathbb{R}, X_{0}^{\prime}\right)
$$


is causal, and its derivative $\mathbf{D}^{(5)}$ is integrable, then $(\mathbf{u}, \theta, \varphi, \phi, \lambda)^{\top} \in C(\mathbb{R}, X)$ is casual, and there holds the estimate

$$
\begin{gathered}
\|\left((\mathbf{u}, \theta, \varphi, \phi, \lambda)^{\top}(t)\left\|_{X} \leq c_{1 / 2} \frac{t^{1+1 / 2}}{(1+t)^{1 / 2}} \max \left\{1, t^{6+1 / 2}\right\} \int_{0}^{t}\right\|\left(\mathcal{P}_{2} \mathbf{D}^{(3)}\right)(\tau) \|_{X^{\prime}} d \tau,\right. \\
\left(\mathcal{P}_{2} \mathbf{D}\right)(t):=\mathbf{D}+2 \dot{\mathbf{D}}+\ddot{\mathbf{D}} .
\end{gathered}
$$

for some constant $c_{1 / 2}>0$.

In view of (25) and the inverse of $\mathbb{A}(s)$, we see that $\widehat{\mathbf{u}}, \widehat{\theta}, \widehat{\varphi}$ and $\widehat{p}$ are simply solutions of the following system

$$
(\widehat{\mathbf{u}}, \widehat{\theta}, \widehat{\varphi}, \widehat{p})^{\top}=A_{2}(s) \circ \mathbb{A}^{-1}(s)\left(\widehat{d_{1}}, \widehat{d_{2}}, \widehat{d_{3}}, \widehat{d}_{4}, 0\right)^{\top},
$$

where

$$
A_{2}(s)=\left(\begin{array}{ccccc}
1 & 0 & 0 & 0 & 0 \\
0 & 1 & 0 & 0 & 0 \\
0 & 0 & 1 & 0 & 0 \\
0 & 0 & 0 & D(s) & -S(s)
\end{array}\right) .
$$

As a consequence of Theorem $3, A_{2}(s) \circ \mathbb{A}^{-1}(s)$ belongs to the class $\mathcal{A}\left(3+1 / 2, \mathcal{B}\left(X_{0}^{\prime}, \mathbb{H}\right)\right)$. However, we may also compute the index of the matrix of operators $A_{2}(s)$. For $\hat{\phi} \in$ $H^{1 / 2}(\Gamma)$, let $\widehat{u}=D(s) \phi \in \mathbb{R}^{3} \backslash \Gamma$, then

$$
\begin{aligned}
\sigma\|\widehat{u}\|_{|s|, \mathbb{R}^{3} \backslash \Gamma}^{2} & =\operatorname{Re}\left(\bar{s}\langle W \widehat{\phi}, \overline{\hat{\phi}}\rangle_{\Gamma}\right) \\
& \leq|s|\|W \widehat{\phi}\|_{H^{-1 / 2}(\Gamma)}\|\widehat{\phi}\|_{H^{1 / 2}(\Gamma)} \\
& \leq c_{1}\left(\frac{|s|}{\underline{\sigma}}\right)^{1 / 2}|s|\|\widehat{u}\|_{|s|, \mathbb{R}^{3} \backslash \Gamma}\|\widehat{\phi}\|_{H^{1 / 2}(\Gamma)}
\end{aligned}
$$

Hence, from (35), we obtain:

$$
\|D(s) \widehat{\phi}\|_{H^{1}\left(\mathbb{R}^{3} \backslash \Gamma\right)} \leq c_{1} \frac{|s|^{3 / 2}}{\sigma \underline{\sigma}^{3 / 2}}\|\widehat{\phi}\|_{H^{1 / 2}(\Gamma)},
$$

which implies $D(s) \in \mathcal{A}\left(3 / 2, \mathcal{B}\left(H^{1 / 2}(\Gamma), H^{1}\left(\mathbb{R}^{3} \backslash \Gamma\right)\right)\right.$. Similarly, for $\widehat{\lambda} \in H^{-1 / 2}(\Gamma)$, if we set $\widehat{u}=S(s) \widehat{\lambda}$ in $\mathbb{R}^{3} \backslash \Gamma$, then we may show that

$$
\|S(s) \widehat{\lambda}\|_{H^{1}\left(\mathbb{R}^{3} \backslash \Gamma\right)} \leq c_{2} \frac{|s|}{\sigma \underline{\sigma}^{2}}\|\widehat{\lambda}\|_{H^{-1 / 2}(\Gamma)} .
$$

That is, $S(s) \in \mathcal{A}\left(1, \mathcal{B}\left(H^{-1 / 2}(\Gamma), H^{1}\left(\mathbb{R}^{3} \backslash \Gamma\right)\right)\right.$, and, hence

$$
\left\|A_{2}(s)\right\|_{X, \mathbb{H}} \leq c_{3} \frac{|s|^{1+1 / 2}}{\sigma \underline{\sigma}^{2+1 / 2}} .
$$

Following [18], if we apply the composition rule and make use of the estimate of $\mathbb{A}^{-1}(s)$ in Equation (46), then we find the matrices of the operators in (51) ended with an index $\mu=(1+1 / 2)+(3+1 / 2)=5$. However, this only gives an upper bound for the actual index of $A_{2}(s) \circ \mathbb{A}^{-1}(s)$ in Equation (51). 


\section{Concluding Remarks}

A few remarks should be in order. This paper deals with a time-dependent wavethermopiezoelectric structure interaction problem by the time-dependent boundary-field equation approach. With the help of a appropriate scaling factor $Z(s)$ in (41), we are able to establish the existence and uniqueness of the solutions to the problem. For simplicity, in this paper, we only impose natural boundary conditions for the corresponding partial differential equations involved in the interior domain $\Omega$. Clearly, one may also impose mixed boundary conditions. Moreover, the results presented in this communication generalize those presented in [22] for elastic-acoustic interactions, [24] for acoustic-piezoelectric interactions, and [23] for acoustic-thermoelastic interactions, since all of those results can be recovered from the ones in this communication by setting to zero selected entries of the piezoelectric tensor, or thermal consants. Moreover, the present work complements the recent articles [41,42], where boundary integral equations of the first kind are studied for the dynamic thermo-elastic equations.

These results can be used to numerically simulate wave-structure interactions by using the nowadays well-known convolution quadrature (CQ) method. Numerical experiments that are based on QC for the special cases of the wave-structure interactions listed above are available in [21-24,43]. The numerical treatment for the operators in the present paper will be reported in a separate communication.

Author Contributions: The contributions of both authors G.C.H. and T.S.-V. of the this research article are equal from the mathematical point of view. Conceptualization, G.C.H and T.S.-V.; methodology, G.C.H. and T.S.-V.; formal analysis, G.C.H. and T.S.-V.; investigation, G.C.H. and T.S.-V.; writingoriginal draft preparation, G.C.H.; writing—review and editing, T.S.-V. All authors have read and agreed to the published version of the manuscript.

Funding: This research received no external funding.

Conflicts of Interest: The authors declare no conflict of interest.

\section{References}

1. Hamdi, M.A.; Jean, P. A mixed functional for the numerical resolution of wave-structure interaction problems. In Aero- and Hydro-Acoustic IUTAM Symposium; Comte-Bellot, G., William, J.E., Eds.; Springer: Berlin, Germany, 1985; pp. $269-276$.

2. Hsiao, G.C.; Kleinman, R.E.; Schuetz, L.S. On variational formulations of boundary value problems for fluid-solid interactions. In Elastic Wave Propagation I.T.U.A.M. -I.U.P.A.P. Symposium; McCarthy, M.F., Hayes, M.A., Eds.; Elsevier Science: Amsterdam, The Netherland, 1989; pp. 321-326.

3. Amini, S.; Harris, P.J. Boundary element and finite element methods for the coupled fluid interaction problem In BEM X; Brebbia, C.A., Ed.; Springer: Berlin, Germany, 1988; pp. 509-520.

4. Bielak, J.; MacCamy, R.C. Symmetric finite element and boundary integral coupling methods for fluid-solid interaction I. Quart. J. Appl. Math. 1991, 49, 107-119. [CrossRef]

5. Hsiao, G.C. On the boundary-field equation methods for fluif-structure In Problems and Methods in Mathematical Physics; TeubnerTexte zur Mathematik, Band 134, B. G.; Jentsch, L., Tröltzsch, F., Eds.; Teubner Veriagsgesellschaft: Stuttgart, Germany, 1994; pp. 79-88.

6. Luke, C.J.; Martin, P.A. Fluid-solid interaction: acoustic scattering by a smooth elastic obstacle. SIAM J. Appl. Math. 1995, 55, 904-922. [CrossRef]

7. Schenk, H.A.; Benthien, G.W. The application of a coupled finite-element boundary-element technique to large-scale structure acoustic problems. In Proceedings of the Eleventh International Conference on Boundary Element Methods, Cambridge, MA, USA, August 1989; Volume 2, pp. 309-318.

8. Elschner, J.; Hsiao, G.C.; Rathsfed, A. On the direct and inverse problems in fluid-structural interaction. In Proceedings of the ICIAM 2007 Proceedings in Applied Mathematics and Mechanics, Zürich, Switzerland, 16-20 July 2007; Volume 7, pp. 1130501-1130502.

9. Elschner, J.; Hsiao, G.C.; Rathsfed, A. An inverse problem for fluid-solid interaction. Inverse Probl. Imag. 2008, 2, 83-120 . [CrossRef]

10. Hsiao, G.C.; Nigam, N. A transmission problem for fluid-structure interaction in the exterior of a thin domain. Adv. Differ. Eq. 2003, 8, 1281-1318.

11. Çakoni, F.; Hsiao, G.C. Mathematical model of the Interaction problem between electromagnetic field and elastic body. In Acoustics, Mechanics, and the Related Topics of Mathematical Analysis; Wirgin, A., Ed.; World Scientific Publishing Co.: Hoboken, NJ, USA, 2002; pp. 48-54. 
12. Gatica, G.N.; Hsiao, G.C.; Meddahi, S. A coupled mixed finite element method for interaction problem between electromagnetic filed and elastic body. SIAM J. Num. Anal. 2010, 48, 1338-1368. [CrossRef]

13. Domínguez, S.; Nigam, N.; Sun, J. Revisiting the Jones Eigenproblem in fluid-structure Interaction. SIAM J. Appl. Math. 2019, 79, 2385-2408. [CrossRef]

14. Hsiao, G.C.; Kleinman, R.E.; Roach, G.F. Weak solutions of fluid-solid interaction problems. Math. Nachr. 2000, 218, 139-163. [CrossRef]

15. Schenck, H.A. Improved integral formulation for acoustic radiation problem. J. Acoust. Soc. Am. 1968, 44, 41-48. [CrossRef]

16. Burton, A.J.; Miller, G.F. The application of integral equation methods to the numerical solution of some exterior boundary-Value problems. Proc. R. Soc. London Ser. A 1971, 323, 201-210.

17. Von Estorff, O.; Antes, H. On FEM-BEM coupling for fluid-structure interaction analysis in the time domain. Int. J. Numer. Meth. Eng. 1991, 31, 1151-1168. [CrossRef]

18. Laliena, A.R.; Sayas, F.-J. Theoretical aspects of the application of convolution quadrature to scattering off acoustic waves. Numer. Math. 2009, 11, 637-678. [CrossRef]

19. Lubich, C.H. On the multistep time discretization of linear initial-boundary value problems and their boundary integral equations. Numer. Math. 1994, 67, 365-389. [CrossRef]

20. Lubich, C.H.; Schneider, R. Time discretization of parabolic boundary integral equations. Numer. Math. 1992, 63, 455-481. [CrossRef]

21. Hassell, M.E.; Qiu, T.; Sánchez-Vizuet, T.; Sayas, F.-J. A new and improved analysis of the time domain boundary integral operators for acoustics. J. Integral Eq. Appl. 2017, 29, 107-136. [CrossRef]

22. Hsiao, G.C.; Sánchez-Vizuet, T.; Weinacht, R.J. Boundary and coupled boundary-finite element methods for transient wavestructure interaction. IMA J. Num. Anal. 2016, 37, 237-265. [CrossRef]

23. Hsiao, G.C.; Sánchez-Vizuet, T.; Weinacht, R.J. A time-dependent fluid-thermoelastic solid Interaction. IMA J. Num. Anal. 2018, 39, 924-956. [CrossRef]

24. Sánchez-Vizuet, T.; Sayas, F.S. Symmetric boundary-finite element discretization of time dependent acoustic scattering by elastic obstacles with piezoelectric behavior. J. Sci. Comput. 2017, 70, 1290-1315. [CrossRef]

25. Acheson, A.J. Elementary Fluid Dynamics. In Oxford Applied Mathematics and Computing Science Series; Clarendon Press: Oxford, UK, 1990.

26. Serrin, J. Mathematical Principles of Classical Fluid Mechanics. In Handbuch der Physik; Springer: Berlin, Germany, 1959; Volume 8, pp. 3-26.

27. Mindlin, R.D. On the equations of motion of piezoelectric crystals. In Problems of Continuous Media; SIAM: Philadelphia, PA, USA, 1961.

28. Kupradze, V.D. Three-dimensional Problems of the Mathematical Theory of Elasticity and Thermoelasticity, North-Holland Series. In Applied Mathematics and Mechanics; North-Holland Publishing Company: Amsterdam, The Netherlands, $1979 ;$; . 164.

29. Nowacki, W. Some general theorems of thermopiezoelectricity. J. Therm. Stres. 1978, 1, 171-182. [CrossRef]

30. Nowacki, W. Electromagnetic Interaction in Elastic Solids. In International Centre for Mechanical Sciences; Parkus, H., Ed.; Springer: Wien, Austria, 1979.

31. Hsiao, G.C.; Weinacht, R.J. A representation formula for the wave equation revoted. Appl. Anal. Int. J. 2012, 91, 371-380. [CrossRef]

32. Hsiao, G.C.; Weinacht, R.J. Transparent boundary conditions for the wave equation-A Kirchhoff point of view. Math. Meth. Appl. Sci. 2013, 36, 2011-2017. [CrossRef]

33. Bamberger, T.; Ha Duong, T. Formulation Variationnelle Espace-Temps pour le Calcul par Potentiel Retard'e de la Diffraction of d'une Onde Acoustique (I). Math. Meth. Appl. Sci. 1986, 8, 405-435.

34. Bamberger, T.; Ha Duong, T. Formulation Variationnelle pour le Calcul de la Diffraction of d'une Onde Acoustique par une Surface Rigide. Math. Meth. Appl. Sci. 1986, 8, 598-608.

35. Costabel, M. Time-dependent problems with the boundary integral equation method, Fundamentals. In Encyclopedia of Computational Mechanics; John Wiley \& Sons, Ltd.: Hoboken, NJ, USA, 2004; Volume 1, pp. 703-721, Chapter 25.

36. Hsiao, G.C.; Wendland, W.L. Boundary Integral Equations. In Applied Mathematical Sciences; Springer: Berlin, Germany, 2008; p. 164

37. Sayas, F.-J. Retarded Potentials and Time Domain Boundary Integral Equations: A Road-Map. In Computational Mathematics; Springer: Berlin, Germany, 2016; p. 50.

38. Fichera, G. Existence theorems in elasticity theory. In Handbuch der Physik; Springer: Berlin, Germany, 1972; Volume 2, pp. 347-389.

39. Hsiao, G.C.; Sayas, F.-J.; Weinacht, R.J. Time-Dependent fluid-structure Interaction. Math. Meth. Appl. Sci. 2017, 40, 486-500. [CrossRef]

40. Sayas, F.-J. Retarded Potentials and Time Domain Boundary Integral Equations: A Road-Map. Available online: https://teampancho.github.io/documents/ERRATA.pdf (accessed on 10 February 2021).

41. Hsiao, G.C.; Sánchez-Vizuet, T. Boundary integral formulations for transient linear thermoelasticity with combined-type boundary conditions. arXiv 2020, arxiv:2010.04909. 
42. Hsiao, G.C.; Sánchez-Vizuet, T. Time-domain boundary integral methods in linear thermoelasticity; Dedicated to the memory of Francisco-Javier Sayas. IMA J. Num. Anal. 2020, 52, 2463-2490.

43. Brown, T.S.; Sánchez-Vizuet, T.; Sayas, F.-J. Evolution of a semidiscrete system modeling the scattering of acoustic waves by a piezoelectric solid. ESAIM: Math. Model. Numer. Anal. (M2AN) 2018, 52, 423-455. [CrossRef] 